\title{
Analysis of GeV-band $\gamma$-ray emission from supernova remnant RX J1713.7-3946
}

\author{
S. Federici ${ }^{1}$, M. Pohl ${ }^{1,2}$, I. Telezhinsky ${ }^{1,2}$, A. Wilhelm ${ }^{1,2}$, and V. V. Dwarkadas ${ }^{3}$ \\ 1 DESY Zeuthen Platanenallee 6, 15738 Zeuthen, Germany \\ e-mail: marpohl@uni-potsdam.de, igor.telezhinsky@desy.de \\ 2 Institute of Physics and Astronomy, University of Potsdam, Karl-Liebknecht-Strasse 24/25, 14476 Potsdam, Germany \\ 3 University of Chicago, Department of Astronomy\& Astrophysics, 5640 S Ellis Ave, TAAC 55, Chicago, IL 60637, USA
}

Received 9 September 2014 / Accepted 22 February 2015

\section{ABSTRACT}

\begin{abstract}
Context. RX J1713.7-3946 is the brightest shell-type supernova remnant (SNR) of the TeV $\gamma$-ray sky. Earlier Fermi-LAT results on low energy $\gamma$-ray emission suggested that, despite large uncertainties in the background determination, the spectrum is inconsistent with a hadronic origin.

Aims. We update the GeV-band spectra using improved estimates for the diffuse Galactic $\gamma$-ray emission and more than double the volume of data. We further investigate the viability of hadronic emission models for RX J1713.7-3946.

Methods. We produced a high-resolution map of the diffuse Galactic $\gamma$-ray background corrected for the HI self-absorption and used it in the analysis of more than five years worth of Fermi-LAT data. We used hydrodynamic scaling relations and a kinetic transport equation to calculate the acceleration and propagation of cosmic rays in SNR. We then determined spectra of hadronic $\gamma$-ray emission from RX J1713.7-3946, separately for the SNR interior and the cosmic-ray precursor region of the forward shock, and computed flux variations that would allow us to test the model with observations.

Results. We find that RX J1713.7-3946 is now detected by Fermi-LAT with very high statistical significance, and the source morphology is best described by that seen in the TeV band. The measured spectrum of RX J1713.7-3946 is hard with index $\gamma=1.53 \pm 0.07$, and the integral flux above $500 \mathrm{MeV}$ is $F=(5.5 \pm 1.1) \times 10^{-9}$ photons $\mathrm{cm}^{-2} \mathrm{~s}^{-1}$. We demonstrate that scenarios based on hadronic emission from the cosmic-ray precursor region are acceptable for RX J1713.7-3946, and we predict a secular flux increase at a few hundred $\mathrm{GeV}$ at the level of around $15 \%$ over ten years, which may be detectable with the upcoming Cherenkov Telescope Array (CTA) observatory.
\end{abstract}

Key words. astroparticle physics - cosmic rays - ISM: supernova remnants - gamma rays: ISM

\section{Introduction}

RX J1713.7-3946, also known as G347.3-0.5, is a young $\left(t_{\mathrm{SNR}} \simeq 1600 \mathrm{yr}\right)$ shell-type supernova remnant $(\mathrm{SNR})$ located in the Galactic plane within the tail of the constellation Scorpius. This SNR was discovered in 1996 during the ROSAT X-rays all-sky survey (Pfeffermann \& Aschenbach 1996). Its shape is slightly elliptical with a maximum extent of $70^{\prime}$, and it exhibits bright X-ray emission predominantly from the western edge of the shell. The assumed distance $d_{\mathrm{SNR}} \simeq 1 \mathrm{kpc}$ implies a shell radius $r_{\mathrm{SNR}} \simeq 8.7 \mathrm{pc}$. The remnant contains an X-ray point-like source whose properties are similar to central compact objects in other SNRs, thus suggesting RX J1713.7-3946 is the remnant of a core-collapse supernova.

Observations with the Japanese ASCA satellite (Koyama et al. 1997) showed a featureless X-ray spectrum in the northwest shell of the remnant, clearly indicating nonthermal emission. Two years later, new ASCA observations (Slane et al. 1999) confirmed the absence of line emission everywhere in the remnant and showed that all parts of the remnant had power laws with indices between $\sim 2.2$ and 2.4. More recent studies of the remnant conducted with Chandra (Uchiyama et al. 2003) and XMM-Newton (Cassam-Chenaï et al. 2004) satellites clearly showed small-scale spatial variations of the photon index ranging from 1.8 to 2.6, which are tracers of the acceleration and propagation history of recently accelerated electrons (Rettig \& Pohl 2012)

At higher energies two competing radiation processes complicate the interpretation of the emission from RX J1713.7 3946. In the leptonic scenario, $\gamma$-rays are produced by electrons via inverse Compton scattering, and in the hadronic scenario $\gamma$-rays are due to $\pi^{0}$-decay from proton-proton interactions. Both scenarios can produce similar fluxes in the $\mathrm{GeV}-\mathrm{TeV}$ energy range.

The HESS telescope array detected RX J1713.7-3946 at the TeV-scale (Aharonian et al. 2004) and showed a similarity between the X-ray and TeV-band morphologies. No decisive conclusion was drawn for the particle population responsible for the emission, although the hadronic scenario was favored.

The Large Area Telescope (LAT; Atwood et al. 2009), the principal instrument on board NASA's Fermi $\gamma$-ray satellite, measured an extended $\mathrm{GeV} \gamma$-ray emission coincident with the position of RX J1713.7-3946 (Abdo et al. 2011). The spectral analysis found a very hard spectral photon index $(\gamma \simeq 1.5)$, which was well in agreement with a leptonic scenario. Nonetheless, this conclusion did not exclude that protons are accelerated in this SNR.

In this paper, we reanalyze Fermi-LAT data that have more than doubled in exposure since the original publication. Moreover, we develop dedicated tools to produce highresolution background maps that take HI self-absorption into 
account. Our background modeling permits determining the key parameters of $\gamma$-ray emission from RX J1713.7-3946, such as spectral index, flux and morphology, with reduced systematic uncertainty. Next, we present a model for hadronic $\gamma$-ray emission from the cosmic-ray precursor of the forward shock of RX J1713.7-3946, which is in principle viable, and, what is more important, we propose a way to verify the model. Based firstly on analytic estimates and then on more detailed calculations we predict a $\gamma$-ray flux increase at the level of $15 \%$ on a timescale of ten years. We suggest this flux variation might be detectable by Cherenkov Telescope Array (CTA; Acharya et al. 2013).

\section{Analysis and results}

\subsection{LAT data}

The LAT is a pair-conversion telescope designed to detect photons from $\sim 20 \mathrm{MeV}$ to $300 \mathrm{GeV}$. A detailed description of the LAT instrument is given in Atwood et al. (2009). The data used in this study come from observations over a period of five years, from August 8, 2008 to August 13, 2013. Data analysis is performed with public analysis tools developed by the LAT team, using the post-launch P7v6 data selection with the appropriate instrument response functions. To minimize the spillover of atmospheric $\gamma$-rays from the Earth limb, a zenith angle cut of $100^{\circ}$ is applied together with a method of correcting the exposure for the zenith cut itself. The method chosen consists in excluding time intervals where any part of the region of interest (ROI) is beyond the zenith-angle limit. Furthermore, data are not taken into consideration while the observatory is transiting the South Atlantic Anomaly (SAA) or when the rocking angle exceeds $52^{\circ}$.

In the analysis only photons with reconstructed energy greater than $500 \mathrm{MeV}$, for which the $68 \%$-containment radius of the PSF is narrower than $\sim 1.5^{\circ}$, are used. The choice of selecting events above $500 \mathrm{MeV}$ is motivated by the earlier published spectrum and by the broad PSF at low energy. The broadening may in fact affect the analysis by leading to systematic problems of source confusion in any densely populated region of the Galactic plane.

The data analysis is done using the publicly available FermiLAT ScienceTools version 9.31.1. In particular, spatial and spectral analyses are performed with dedicated scripts based on the Python likelihood tools that expand upon the command line tools provided with the Fermi Science Tools package.

Since the analysis is performed over a large number of observed events, the binned likelihood analysis is preferred. The ROI is a square region measuring $28^{\circ}$ on a side and centered on $\alpha=258.39^{\circ}$ and $\delta=-39.76^{\circ}$ (J2000), the nominal position of RX J1713.7-3946. The angular binning is chosen to be $0.125^{\circ}$ per pixel in stereographic projection to match the resolution of the corrected diffuse $\gamma$-ray background. To preserve accuracy in the likelihood analysis, 30 energy bins are chosen, which allows us to accommodate rapid variations in the effective area with decreasing energy below $\sim 1 \mathrm{GeV}$.

The $\gamma$-ray background is modeled in an acceptance region, called the source region, which is $5^{\circ}$ larger than the ROI. This accounts for sources that lie just outside the data region, but whose photons spilled into the data set. The background model includes 174 point-like sources and 2 extended sources listed in the 2nd Fermi-LAT catalog (Nolan et al. 2012). The source 2FGL J1712.4-3941 is not considered in the model because it is spatially coincident with RX J1713.7-3946. Furthermore,

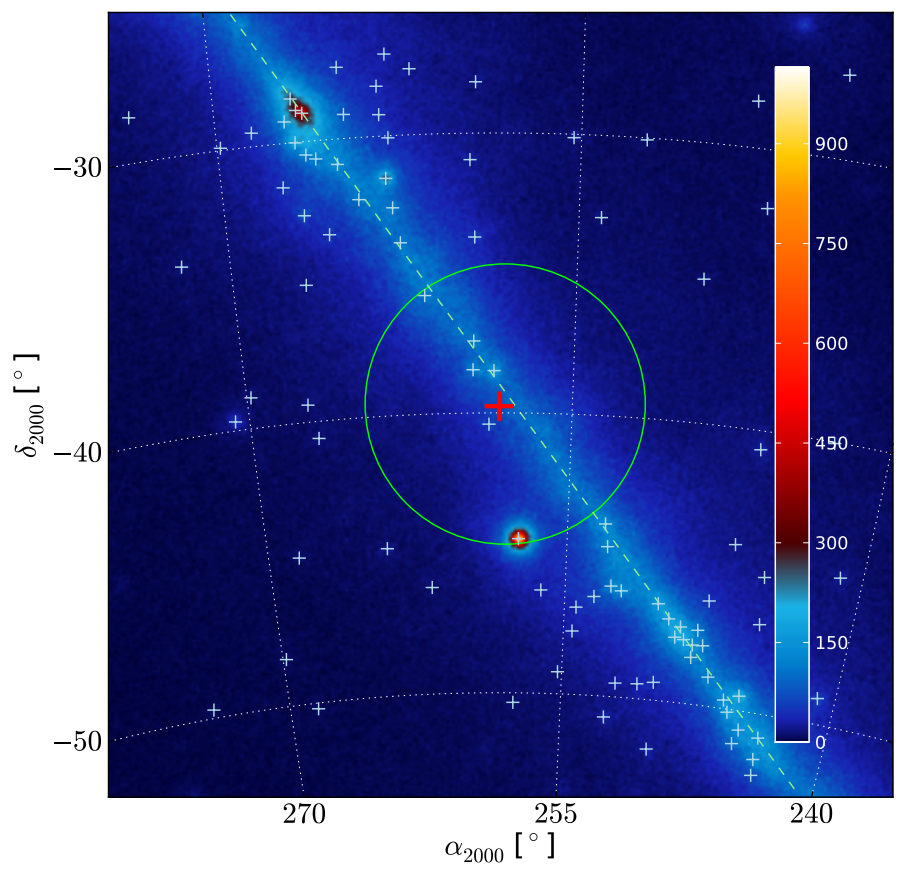

Fig. 1. Counts map of the region of interest (ROI) used in this work. Sources of the 2nd Fermi-LAT catalog are marked by light blue crosses. The green dashed line denotes the Galactic plane and the red cross represents the nominal position of RX J1713.7-3946. During the fit procedure the parameters of the sources inside the green circular region of $5^{\circ}$ radius are free to vary.

we include an isotropic template ${ }^{1}$ that accounts for extragalactic emission and residual cosmic-ray contamination. Finally we use a modified template for Galactic diffuse $\gamma$-ray emission that shall be described in the next section.

Amongst the point-like and extended sources, 105 lie inside the ROI and most of them are modeled with power-law energy spectra. The spatial and spectral parameters of sources located outside a circular region of $5^{\circ}$ radius centered at the nominal position of RX J1713.7-3946 are kept fixed at the values given in the catalog. Inside this region, the spectral parameters of seven sources along with the normalization of the diffuse components, are permitted to freely vary. Thus, the final set of free parameters is reduced to 20 , a number that allows the fit to converge. Figure 1 shows the ROI with 105 sources of the 2nd Fermi-LAT catalog and the circular region of $5^{\circ}$.

\subsection{The HISA contribution to the diffuse Galactic $\gamma$-ray emission}

The likelihood analysis of data from Fermi-LAT requires templates for an isotropic component, intended to cover extragalactic emission as well as residual instrumental background, and diffuse Galactic $\gamma$-rays. The latter is based on infrared tracers and spectral line information on atomic hydrogen and carbon monoxide as tracer of molecular gas.

While the absolute normalization of the templates is adjustable in the likelihood optimization, its angular structure is not. If an extended source is to be analyzed, a poor representation of the diffuse background will lead to systematic errors in the reconstructed morphology and possibly a significant offset in the derived spectrum of the source.

Available from the Fermi Science Support Centre. 
A major source of uncertainty is $\mathrm{H} \mathrm{I}$ absorption, to which a first-order opacity correction is often applied by assuming a uniform spin temperature, $T_{\mathrm{s}}$. In terms of the brightness temperature, $T_{\mathrm{b}}$, and for low continuum intensity, a correction factor can be derived as

$\epsilon=-\ln \left(1-\frac{T_{\mathrm{b}}}{T_{\mathrm{s}}}\right) \frac{T_{\mathrm{s}}}{T_{\mathrm{b}}}$

where one typically uses values of $T_{\mathrm{s}}$ in the range $120-150 \mathrm{~K}$. While this method has some merit, high-resolution H I surveys have recently indicated the existence of large variations in spin temperature from one cloud to the next. Small-scale selfabsorption features (HISA, as for H I self-absorption) were identified that are caused by relatively cold $(\sim 50 \mathrm{~K})$ gas clouds that are located in front of much warmer gas and sources of continuum emission. Their effect on spectral line data goes beyond a simple correction as in Eq. (1) on account of both its absorption effect on the baseline continuum spectrum and the substantial gas mass that can be carried by the compact clouds.

We have used data from the Southern Galactic Plane Survey (SGPS; McClure-Griffiths et al. 2005) to refine the standard model of Galactic diffuse emission ${ }^{2}$ to a higher intrinsic angular resolution than is provided in single-dish H I surveys and to correct for HISA using the algorithm described in Gibson et al. (2005).

The idea behind Gibson's method is to iteratively remove large-scale spectral and spatial emission structures from $\mathrm{H}$ I data and to flag the small-scale negative residuals as self-absorption features. The additional optical depth imposed by the cold $\mathrm{HI}$ absorber is estimated using the brightness-temperature difference between a line of sight through the absorber and other lines of sight in the vicinity, assuming most of the continuum signal is produced behind the structure. The density and mass of the absorber are calculated assuming pressure equilibrium. Further details on the analysis can be found in Gibson's paper.

The additional H I column density derived for the absorber is then combined with that associated with the absorption correction to the original line signal. For both we calculate the location on the line of sight using the method of Pohl et al. (2008). We then use the $\gamma$-ray emissivity derived with the GALPROP $\operatorname{code}^{3}$, version 54, with best-fit parameters given in Strong et al. (2010), to compute the expected $\gamma$-ray intensity from the additional H I column density, which we found in the HISA search. Finally, we correct the standard templates of diffuse Galactic $\gamma$-ray emission for the additional $\gamma$-ray signal.

As high-resolution gas maps are available only for a narrow strip along the Galactic plane, they are combined with the lowresolution maps of the Leiden-Argentina-Bonn survey (Kalberla et al. 2005), and the composite map is sampled with a pixel size of $0.125^{\circ}$. At the location of RX J1713.7-3946 we thus have an accurate sampling of structure in the diffuse Galactic $\gamma$-ray emission on scales commensurate with the angular resolution of LAT, whereas off the plane the intrinsic resolution is that of the standard Galactic-emission template available at the Fermi Science Support Center.

To study the impact of the correction for the HISA on the diffuse Galactic emission (DGE) two binned likelihood analyses are performed, one with the standard Fermi-LAT DGE template and one with the corrected DGE template. The detailed setup of the likelihood fit, used for the standard Science Tool gtlike,

\footnotetext{
2 Publicly available at the Fermi Science Support Center.

3 http://galprop.stanford.edu/
}

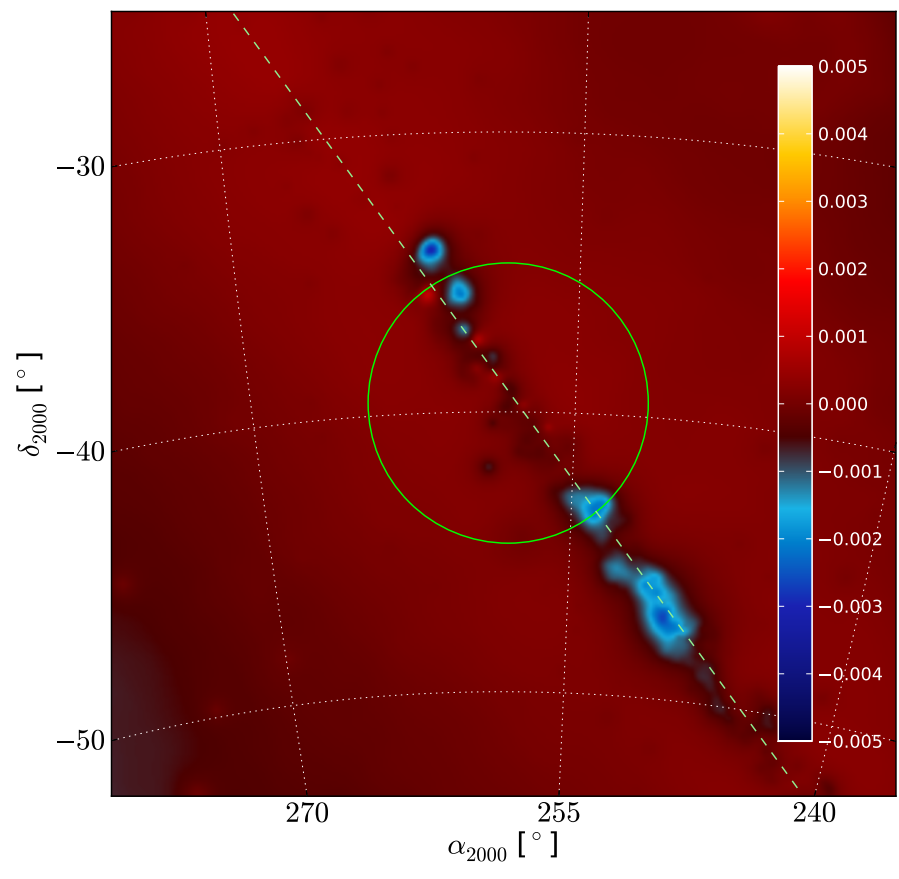

Fig. 2. Map of the difference in fit residuals of the revised and the standard template of Galactic emission.

is identical for both analyses. The two likelihood fits are performed, assuming as a spatial template for RX J1713.7-3946, a uniform disk of $0.5^{\circ}$ radius. The fits are performed in the energy range spanning from $500 \mathrm{MeV}$ to $300 \mathrm{GeV}$ and indicate that the revised model of Galactic $\gamma$-ray emission in the ROI is favored with a significance of $\sim 4 \sigma$.

Figure 2 shows the difference in the best-fit model maps of the two cases, $\left[m_{1}-m_{2}\right] / \sqrt{m_{2}}$, where $m_{1}$ and $m_{2}$ refer to the standard and the revised templates, respectively. To be noted from the figure are the localized negative features, that correspond to HISA corrections. At the location of RX J1713.7-3946, no significant absorption feature was found, and, as we have tested, using the revised background model changes the best-fit flux from RX J1713.7-3946 by only about $1 \%$.

\subsection{Position and spatial extension of $R X$ J1713.7-3946}

After the first two years of science operation of Fermi, LAT has established a firm detection of $\gamma$-ray emission from RX J1713.7-3946 with statistical significance of $\sim 9 \sigma$. Now, three years later, the accumulated exposure has more than doubled, and it is expected that systematic uncertainties arising from, e.g., the spatial distribution of emission coincident with the SNR, have an impact on the reconstructed $\gamma$-ray spectrum. Before determining the spectrum, we therefore first need to find the localization and spatial distribution of emission from RX J1713.7-3946.

To find the center of gravity of events from RX J1713.73946, we construct a map of test statistic (TS; Mattox et al. 1996), defined as logarithmic improvement in the likelihood function $L$,

$\mathrm{TS}=2\left(\ln L-\ln L_{0}\right)=2 \ln \left(\frac{L}{L_{0}}\right)$,

which we achieve by moving a putative point-like source through a grid of locations on a square region. The region measures $\sim 3^{\circ}$ on a side and is centered at the nominal position of 


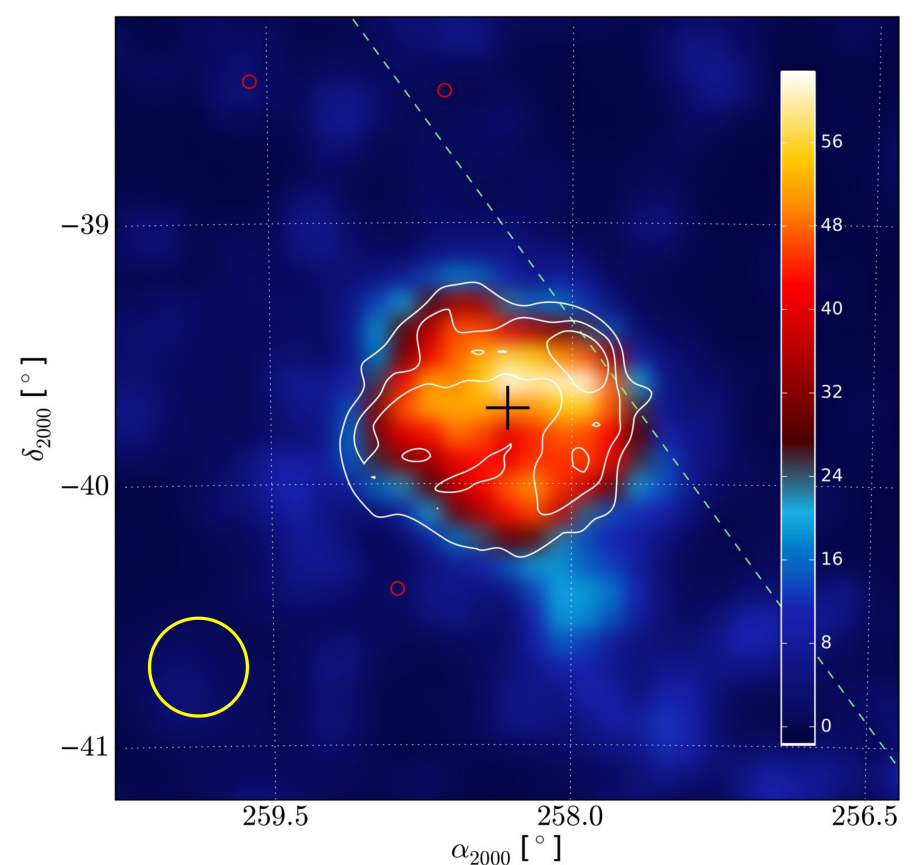

Fig. 3. Map of the test statistic (TS) for a point-like source in the region around RX J1713.7-3946. The black cross denotes the best-fit centroid of a disk model and the yellow circle indicates the energyaveraged $68 \%$ confinement region of the point-spread function. Red circles indicate the position of the second Fermi-LAT catalog sources in the background model. Shown in white are contours of $\gamma$-ray excess counts based on HESS observations, the levels are 25, 50, and 75.

the SNR. At each point of the grid the parameters of the pointlike source are free to vary and those of all background components are fixed at the values found in the previous likelihood analysis (see Sect. 2.2). The resolution of the grid is $0.1^{\circ}$, which implies 961 likelihood analyses. The resulting TS map is shown in Fig. 3. The envelope of signal with TS $\geq 36$ suggests spatially extended emission because a true point source would appear with localization uncertainty corresponding to the energyaveraged width of the point-spread function (PSF, indicated in Fig. 3 by the yellow circle) divided by the detection significance, i.e., with $\lesssim 0.2^{\circ}$ in diameter. Particularly interesting is the local enhancement of the signal in the northwest region of the shell with TS $>49$, which matches well the excess-event density seen with HESS, and is indicated with white intensity contours with levels 25, 50, and 75 .

To find the best position of the source and hence minimize the systematic uncertainties, we then modeled RX J1713.7-3946 as a uniform disk with a radius of $0.5^{\circ}$ instead of a point-like source. The best-fit centroid for the disk model is indicated in Fig. 3 and located at $\alpha=258.32^{\circ}$ and $\delta=-39.71^{\circ}$ in J2000 with an error radius of $0.02^{\circ}$ at the $68 \%$ confidence level, which is about $0.07^{\circ}$ off the nominal coordinates of the source in direction of the Galactic plane. All the following analyses are performed using spatial templates centered at this best-fit position.

To investigate the spatial morphology of the emission associated with RX J1713.7-3946 a number of spatial templates are tested. Five templates are uniform disks with radii ranging from $0.5^{\circ}$ to $0.7^{\circ}$ in steps of $0.05^{\circ}$. An additional template is designed to reflect the TeV-band intensity distribution observed with the HESS telescope. In Fig. 4 we show all these spatial templates after being convolved with the Fermi-LAT PSF. Because of the broadening of the PSF at low energies, the detailed shape and size of the six templates can only be distinguished at high

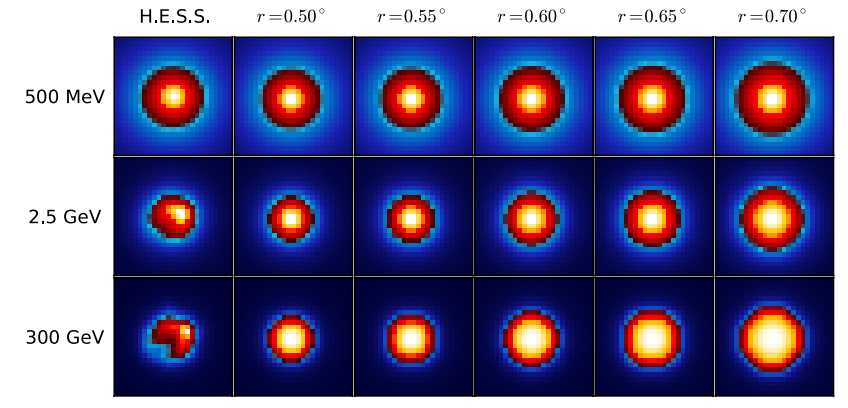

Fig. 4. Spatial templates used to model the emission associated with RX J1713.7-3946. The templates are convolved with the Fermi-LAT PSF and are shown for three different energies.

Table 1. Morphological analysis of the $\gamma$-ray emission associated with RX J1713.7-3946.

\begin{tabular}{lccc}
\hline \hline Morphology & Flux $^{a}$ & Photon index & TS \\
\hline Disk $r=0.50^{\circ}$ & $4.982 \pm 0.916$ & $1.508 \pm 0.075$ & 160 \\
Disk $r=0.55^{\circ}$ & $5.122 \pm 0.941$ & $1.509 \pm 0.075$ & 161 \\
Disk $r=0.60^{\circ}$ & $5.449 \pm 1.069$ & $1.513 \pm 0.075$ & 159 \\
Disk $r=0.65^{\circ}$ & $5.547 \pm 1.052$ & $1.514 \pm 0.075$ & 156 \\
Disk $r=0.70^{\circ}$ & $5.908 \pm 1.216$ & $1.521 \pm 0.076$ & 150 \\
HESS & $5.522 \pm 1.075$ & $1.528 \pm 0.074$ & 163 \\
\hline
\end{tabular}

Notes. ${ }^{(a)}$ The integral flux from RX J1713.7-3946 is calculated over the energy range $500 \mathrm{MeV}-300 \mathrm{GeV}$ and it is given in units of $10^{-9}$ photons $\mathrm{cm}^{-2} \mathrm{~s}^{-1}$.

energies. Table 1 summarizes the best-fit parameters in the likelihood analysis of each template, performed in the energy range from $500 \mathrm{MeV}$ to $300 \mathrm{GeV}$ by optimizing a spectral model of the form

$\frac{\mathrm{d} N}{\mathrm{~d} E}=N_{0}\left(\frac{E}{E_{0}}\right)^{-\gamma}$,

where $N_{0}$ is the prefactor, $E_{0}$ the energy scale, and $\gamma$ is the spectral index.

All models yield a high test statistic $(150 \leq \mathrm{TS} \leq 163)$, about three times that achieved in a point-source fit, implying that the emission is well resolved by the LAT as an extended region. For uniform-disk templates, TS slowly decreases beyond a radius of $0.55^{\circ}$, indicating that this value marks the extent of $\mathrm{GeV}$-scale emission from RX J1713.7-3946. The HESS template fits best, and the TS value for the uniform disk of radius $0.55^{\circ}$ is also acceptable $(\Delta \mathrm{TS}=2)$. As the HESS template does not fit significantly better than the disk of radius $0.5^{\circ}$ that we used to find the centroid position, we see no reason to deviate from the sequence of analysis steps defined a priori, namely first finding the centroid position and then determining the best-fitting template. We thus use the HESS template, centered at $\alpha=258.32^{\circ}$ and $\delta=-39.71^{\circ}$ in $\mathbf{J} 2000$, in the following spectral analysis. In the end, the vagaries in choosing the spatial template and centroid position contribute to the systematic-uncertainty margin that we shall discuss below.

\subsection{Energy spectrum of $R X \mathrm{~J} 1713.7-3946$}

As a first step toward measuring the spectrum of $\gamma$-ray emission from the SNR, we perform a global likelihood analysis using the HESS template as spatial model for RX J1713.7-3946. The fit 


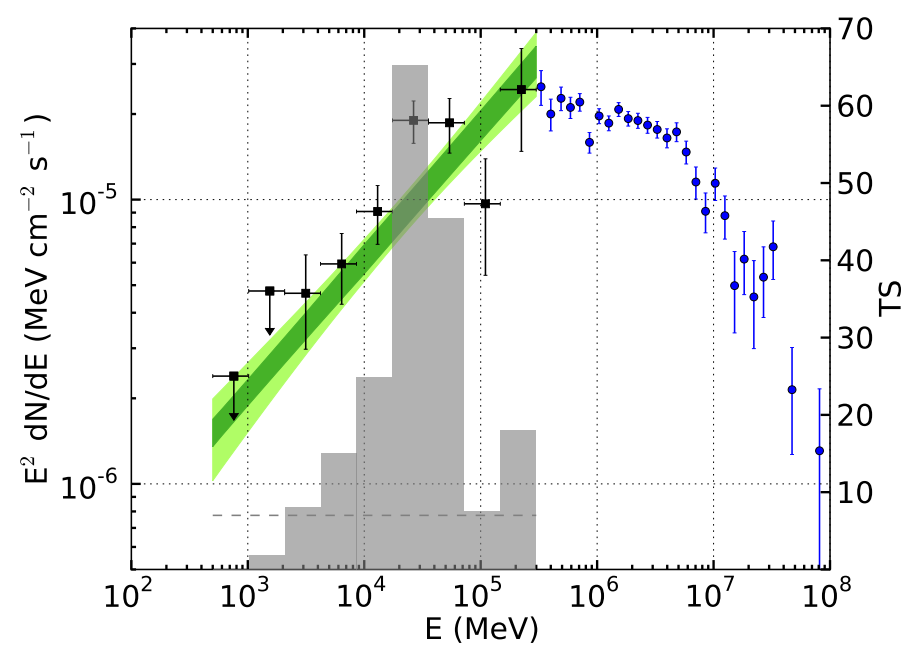

Fig. 5. Broadband $\gamma$-ray spectrum of RX J1713.7-3946 as measured here (black points) and by HESS (blue points). The two bow tie bands denote systematic (dark green) and total (light green) uncertainties of the single power-law fit, the latter assuming that systematic and statistical uncertainties can be added in quadrature. The histogram indicates the TS value for each energy bin. The dotted line is the threshold for setting a $95 \%$ confident level upper limit.

yields a $\gamma=1.53 \pm 0.07$ and the measured integral photon flux above $500 \mathrm{MeV}$ is $F=(5.52 \pm 1.07) \times 10^{-9}$ photons $\mathrm{cm}^{-2} \mathrm{~s}^{-1}$.

In order to obtain a spectral energy distribution (SED) for the SNR, the entire energy range is divided into nine logarithmically spaced energy bins. For each individual bin, a likelihood fit is performed using the HESS template as spatial model for RX J1713.7-3946 and the spectral model and parameters obtained in the global fit (and shown in Table 1). For those energy bins where TS $<7$, a $95 \%$ confidence-level flux upper limit is calculated.

To estimate the amplitude of systematic uncertainties, the data are refitted for several variations of the default model. First, the corrected diffuse-background model is replaced by the standard background model, yielding a $1 \%$ variation in the derived flux. Second, the HESS template is substituted with reasonably fitting uniform-disk templates (radii $0.5^{\circ}, 0.55^{\circ}, 0.6^{\circ}$ ), indicating an uncertainty of $\sim 6 \%$ at $20 \mathrm{GeV}$ increasing to $10 \%$ at $500 \mathrm{MeV}$ and $8 \%$ at $300 \mathrm{GeV}$. For the worst-fitting template, the disk of $0.7^{\circ}$ radius, we find an offset of $10 \%$ at $20 \mathrm{GeV}$. Furthermore, uncertainties due to instrumental effects are also taken into account. The flux measurement in fact depends on the knowledge of the effective collecting area of LAT as a function of energy. The systematic error in the effective area for the P7SOURCE_V6 event class may be quoted as $10 \%$ at $100 \mathrm{MeV}, 5 \%$ at $560 \mathrm{MeV}$, and again $10 \%$ at $10 \mathrm{GeV}$ and above (Ackermann et al. 2012b). All these contributions combined indicate overall systematic uncertainties at the level of about $11 \%$ at $500 \mathrm{MeV}, 12 \%$ at $20 \mathrm{GeV}$, and $13 \%$ at $300 \mathrm{GeV}$.

The spectral data points are given in Table 2, and the resulting energy spectrum of RX J1713.7-3946 is shown in Fig. 5 together with previously published HESS measurements (Aharonian et al. 2011). The two green bands indicate the systematic and the total uncertainties in a fit of a single power law. The nine Fermi-LAT data points (black crosses) only include statistical errors. In the background of the figure, the gray histogram represents the distribution of TS values obtained for each bin of the Fermi-LAT SED. The dotted line at the bottom of the histogram denotes the threshold $(\mathrm{TS}=7)$ for setting a 95\% confidence-level upper limit on the Fermi-LAT
Table 2. Spectral data points of GeV-scale $\gamma$-ray emission associated with RX J1713.7-3946.

\begin{tabular}{lrr}
\hline \hline$E_{\text {mean }}{ }^{a}$ & $E F(E)$ & $\delta[E F(E)]$ \\
\hline $7.59 \times 10^{2}$ & 2.40 & 0.59 \\
$1.55 \times 10^{3}$ & 4.77 & 1.58 \\
$3.14 \times 10^{3}$ & 4.68 & 1.71 \\
$6.40 \times 10^{3}$ & 5.94 & 1.66 \\
$1.30 \times 10^{4}$ & 9.08 & 2.12 \\
$2.65 \times 10^{4}$ & 19.0 & 3.23 \\
$5.40 \times 10^{4}$ & 18.6 & 4.06 \\
$1.10 \times 10^{5}$ & 9.67 & 4.25 \\
$2.24 \times 10^{5}$ & 24.4 & 9.60 \\
\hline
\end{tabular}

Notes. (a) Energies are in $\mathrm{MeV}$ and the $E F(E)$ flux from RX J1713.7-3946 is calculated in units of $10^{-6} \mathrm{MeV} \mathrm{cm}^{-2} \mathrm{~s}^{-1}$.

data points. The physical interpretation of the SED will be discussed in the next section.

\section{Interpretation}

The hard spectrum of the GeV-band emission initially discovered by Abdo et al. (2011) and confirmed here suggests an inverse Compton (IC) origin of the emission. In a simple one-zone scenario for the particle distribution and subsequent emission, it is difficult to accommodate a strong hadronic component. In this section, we point out that more complex scenarios are possible, although they require some degree of fine-tuning. In an analytic assessment and detailed calculations we explore the viability of a hadronic-emission scenario involving radiation from the vicinity of RX J1713.7-3946.

One-zone models of the broadband SED from X-ray (Tanaka et al. 2008) to GeV $\gamma$-ray (Fermi-LAT), and TeV $\gamma$-ray (HESS) energies invariably wash over, e.g., the variations in the X-ray spectrum across the remnant (Sano et al. 2015), which may reflect electron aging and a changing magnetic field (Rettig \& Pohl 2012; Ressler et al. 2014). A few general insights can be gained, though. Stringent constraints on the gas density in RX J1713.7-3946 are given by the absence of a clear detection of thermal X-ray emission (Tanaka et al. 2008), indicating a post-shock gas density of $n_{\mathrm{H}} \lesssim 0.8 \mathrm{~cm}^{-3}$.

Given the low gas density and the $\gamma$-ray brightness of RX J1713.7-3946, hadronic models require a very large energy density in cosmic rays and a very hard particle spectrum starting from lowest energies to reproduce the observed GeV-TeV $\gamma$-ray spectrum. This is difficult to explain with diffusive shock acceleration even in the nonlinear mode. Leptonic models, on the other hand, invariably require an unusually weak magnetic field of amplitude $\sim 10 \mu \mathrm{G}$, implying that electron energy losses are negligible and at odds with theoretical expectations for efficient diffusive shock acceleration, that there is no significant amplification of magnetic field at the shock. Also, the electron spectrum must be moderately soft ( $\alpha \gtrsim 2)$ to simultaneously account for X-ray, Fermi and HESS data. There are more complicated hadronic models, however, which may fit the GeV-scale emission from RX J1713.7-3946. We discuss these models below.

\subsection{Hadronic emission from the SNR interior}

RX J1713.7-3946 was likely produced by a core-collapse supernova. The progenitor star would have emitted a wind that pushed the ambient medium into a dense gas shell at some distance. The gas density inside the wind zone is low, implying a weak 
deceleration of the forward shock as is observed. The high velocity currently measured suggests that the forward shock has not yet reached the wall enclosing the wind-blown cavity.

Inoue et al. (2012) suggested that dense gas clouds survive passage through the forward shock of RX J1713.7-3946. Slow and energy-dependent diffusion of cosmic rays into these clouds would then lead to a hard particle spectrum at the high-density core of the cloud. Gabici \& Aharonian (2014) demonstrate that a fit to the GeV-to-TeV spectrum can be achieved, albeit apparently requiring a mass of $\sim 500 M_{\odot}$ inside the SNR, carried only by massive clouds that GeV-TeV-scale cosmic rays cannot fully penetrate. This is much more than can condense out of the wind of the massive progenitor star of RX J1713.7-3946, and so the scenario would require that pre-existing clouds have been unaffected by the wind. Besides the fact that nonpenetration of clouds is not observed in the $\gamma$-ray spectra of nearby molecular complexes (Ackermann et al. 2012a), we see two difficulties with this scenario.

Firstly, the medium around massive stars tends to be largely homogenized before they explode as core-collapse supernova, owing to photoevaporation and the rocket effect (McKee et al. 1984). Secondly, as the stellar wind streams around dense gas clouds, Kelvin-Helmholtz instabilities, followed by Rayleigh-Taylor instabilities will disrupt and wash off their outer layers, leading to large streams of gas with moderate density $\left(n \approx 1-10 \mathrm{~cm}^{-3}\right)$. Upon contact with the SNR forward shock, these streams of gas would emit intense X-ray emission. One would have to demonstrate that its intensity is compatible with the published upper limits for thermal $\mathrm{X}$ radiation.

A large fraction of the gas clouds and the outer gas shell will thus be located outside of the SNR, where they would be illuminated by the cosmic-ray precursor to the forward shock. We discuss this scenario in the next subsection.

\subsection{Hadronic emission from the cosmic-ray precursor}

The width of the cosmic-ray precursor to astrophysical shocks is strongly energy dependent on account of the momentum dependence of the mean free path for scattering. At a given location, this leads to very hard particle spectra with cutoff at an energy determined by the distance from the shock, the diffusion coefficient, and the shock velocity (Gabici et al. 2009; Ellison \& Bykov 2011; Telezhinsky et al. 2012b). It has been argued before that the escape of cosmic rays from SNRs might lead to strong $\mathrm{TeV}$-band $\gamma$-ray emission from molecular clouds located $10 \mathrm{pc}$ or more from the remnant. In the case of RX J1713.7-3946, the target gas has to be located very close to the forward shock, otherwise we cannot observe intense emission at 50-500 GeV. Consequently, it is illuminated not primarily by so-called runaway cosmic rays that have escaped from the system, but by the cosmic-ray precursor to the forward shock. We do not doubt that it would be possible to find parameters that permit reproducing the observed $\gamma$-ray spectrum of RX J1713.7-3946 within such a scenario. Rather, we predict a gradual but detectable increase in $\gamma$-ray flux over a decade or two that renders this scenario testable. To substantiate our claim, we first present an analytic estimate to illustrate why the signal would grow with time. We then use a more sophisticated model to calculate the time dependence of the $\gamma$-ray spectrum.

\subsubsection{Analytic estimate}

We begin with an analytic model, that despite its simplicity, demonstrates the existence and magnitude of time dependence in the $\gamma$-ray signal from a gas cloud just outside of an SNR. The transport equation for the differential number density of cosmicrays, $N(E, r)$, comprises terms for spatial diffusion and advection, which assuming spherical symmetry can be written as

$$
\begin{aligned}
\frac{\partial N(E, r)}{\partial t} & -\frac{1}{r^{2}} \frac{\partial}{\partial r}\left[r^{2} D(E, r) \frac{\partial N(E, r)}{\partial r}-r^{2} V(r) N(E, r)\right] \\
& +\mathcal{D}_{E} N(E, r)=Q(E, r),
\end{aligned}
$$

where $\mathcal{D}_{E}$ denotes the differential operator in energy. Rewriting Eq. (4) in comoving coordinates anchored on the shock position, $r_{\mathrm{s}}$,

$r \rightarrow \rho=r-r_{\mathrm{s}}$ and $V_{\rho}=V-\dot{r}_{\mathrm{s}}$

and restricting to the vicinity of the forward shock $\left(\rho \ll r_{\mathrm{s}}\right.$ ), we may assume a plane-parallel geometry. As particle acceleration is faster than the hydrodynamical evolution of the SNR, the particle distribution in comoving coordinates can be in good approximation written in the steady-state limit,

$$
-\frac{\partial}{\partial \rho}\left[D(E, \rho) \frac{\partial N(E, \rho)}{\partial \rho}-V_{\rho} N(E, \rho)\right]+\mathcal{D}_{E} N(E, \rho)=Q(E, \rho)
$$

Note that in the shock precursor region sources are absent, i.e., $Q(E, \rho)=0$. The term containing $\mathcal{D}_{E}$ is expected to be small because energy-changing processes operating in the precursor region are very slow compared to the SNR evolution, and so we have to search for homogeneous solutions to the spatial part of the transport equation,

$$
\frac{\partial}{\partial \rho}\left[D(E, \rho) \frac{\partial N(E, \rho)}{\partial \rho}-V_{\rho} N(E, \rho)\right] \simeq 0 .
$$

Generally, there are two solutions to Eq. (7), which are approximately

$N_{1} \simeq \frac{A}{V_{\rho}} \quad N_{2} \simeq C \exp \left(\int^{\rho} \mathrm{d} \rho^{\prime} \frac{V_{\rho}}{D\left(E, \rho^{\prime}\right)}\right)$,

where $A$ and $C$ are constants that are determined by matching the cosmic-ray density at the shock. The plasma flow speed in the precursor region can be somewhat modified by nonlinear shock modification, and so we only know $V_{\rho} \gtrsim-\dot{r}_{\text {s }}$. It is obvious that only $N_{2}$ satisfies the boundary condition $N(\rho=\infty)=0$, therefore it must describe the true cosmic-ray density profile reasonably well.

Now suppose a gas cloud is located at radius $r_{0}$, corresponding to the time-dependent comoving coordinate $\rho_{0}(t)=$ $r_{0}-r_{\mathrm{s}}(t) \simeq r_{0}-\dot{r}_{\mathrm{s}} t$, where the last expression is approximately valid for short time intervals. The gas cloud is illuminated with cosmic rays of differential density,

$N \simeq N_{0}(E) \exp \left(\int^{\rho_{0}} \mathrm{~d} \rho^{\prime} \frac{V_{\rho}}{D\left(E, \rho^{\prime}\right)}\right)$

The timescale on which the cosmic-ray illumination changes can be estimated as

$\tau \simeq \frac{N}{\left|\frac{\partial N}{\partial t}\right|} \simeq \frac{D\left(E, \rho_{0}\right)}{\left|\dot{\rho}_{0} V_{\rho}\right|} \simeq \frac{D\left(E, \rho_{0}\right)}{\dot{r}_{\mathrm{s}}^{2}}$ 
If we scale the diffusion coefficient to the Bohm limit, $D=$ $\eta r_{\mathrm{L}} c / 3$, we find for the timescale of variation

$\tau \simeq(10 \mathrm{yrs}) \eta\left(\frac{E}{10 \mathrm{TeV}}\right)\left(\frac{B}{10 \mu \mathrm{G}}\right)^{-1}\left(\frac{\dot{r}_{\mathrm{s}}}{3000 \mathrm{~km} \mathrm{~s}^{-1}}\right)^{-2}$.

Protons with $10 \mathrm{TeV}$ in kinetic energy produce $\gamma$-rays at energies around $500 \mathrm{GeV}$, at which imaging atmospheric Cherenkov telescope have their peak sensitivity. As long as the diffusion coefficient is not significantly larger than the Bohm limit, we expect observable variations in the $\gamma$-ray flux from bright, young SNRs such as RX J1713.7-3946, in particular if streaming instabilities amplify the magnetic field to amplitudes in excess of $10 \mu \mathrm{G}$.

To be noted is that the variation timescale does not explicitly depend on the distance from the shock. Implicitly, a certain distance dependence arises from the fact that the diffusion coefficient must be evaluated near the location of the gas cloud, not at the shock.

\subsubsection{Detailed modeling}

In this subsection we describe a more detailed model of RX J1713.7-3946 and its high energy (HE) $\gamma$-ray flux variability, which we expect according to the predictions outlined in Sect. 3.2.1. We consider a system consisting of a core-collapse SNR and a shell of dense gas located just outside the remnant. We do not pretend to give a good broadband fit to the data because that would require a sophisticated model of the evolution of, and subsequent emission from, the remnant itself (e.g., Telezhinsky et al. 2013). In any case, the remnant itself is a rather faint source of $\gamma$-ray emission on account of the low-density environment left by the progenitor stellar wind. The hadronic emission is dominated by $\gamma$-rays from the dense shell that is illuminated by high energy cosmic rays in the precursor. Consequently, the overall spectrum is expected to be hard. In low energy (LE) $\gamma$-rays the flux from the SNR and the shell may be comparable because a low gas density near the forward shock is compensated by a high intensity of LE cosmic rays. The flux of HE $\gamma$-rays from the dense shell and its variation depend on the cosmic-ray diffusion coefficient and the current kinematics of the shock, parameters that change little if sophisticated models of SNR are considered. Therefore, the prediction of HE $\gamma$-ray flux variation offered here is robust.

We numerically solve the full transport equation for cosmic rays (4) on a grid that extends from the SNR center to several dozens of SNR radii ahead of the forward shock (FS). The grid extension allows us to account for the escape from the precursor of particles of both species, protons and electrons. We can use either full hydrodynamical simulations or analytic solutions to trace the evolution of the SNR, i.e., the velocity and radius of shocks and the plasma flow profile inside the remnant. A crucial parameter for particle acceleration is the diffusion coefficient. We assume it to be Bohm-like, $D_{\mathrm{B}}=\eta r_{\mathrm{L}} v / 3$, where $\eta=1.0$ (at the shock), $r_{\mathrm{L}}$ is the particle Larmor radius, and $v$ is the particle velocity. Ahead of the shock the diffusion coefficient linearly increases (Bell 1978; Drury 1983) until thirty SNR radii ahead of the shock it reaches $D_{G}=10^{29}(E / 10 \mathrm{GeV})^{1 / 3}(B / 3 \mu \mathrm{G})^{-1 / 3}$, a value typical for the Galactic propagation. Close to the shock diffusion is still Bohm-like, and so a typical cosmic-ray precursor will be established. Further ahead, the probability of escape from the system becomes significant, and particles may leave the system. For further details see Telezhinsky et al. (2012a,b, 2013).

Given that thermal X-rays are not detected, the current number density of gas upstream of the blast wave must be around $0.05 \mathrm{~cm}^{-3}$, much lower than the typical density in the interstellar medium. Therefore, the explosion should have taken place in the wind-blown bubble of the massive progenitor star, and the SN may be classified as a core-collapse event. To reconstruct these parameters we used analytical solutions for the free-expansion stage of SNR expanding into a wind-blown cavity (Truelove \& McKee 1999). The current SNR radius $R \simeq$ $8.65 \mathrm{pc}$ and shock speed $V_{\mathrm{sh}} \simeq 4200 \mathrm{~km} \mathrm{~s}^{-1}$ (for a distance of $1 \mathrm{kpc}$ ) permit a range of parameter values for the massloss rate and the progenitor wind speed. As an example, we assumed for the mass-loss rate $\dot{M} \simeq 10^{-4} M_{\odot}$, for the wind speed $v_{\mathrm{w}} \simeq 50 \mathrm{~km} \mathrm{~s}^{-1}$, and for the explosion energy $E_{\mathrm{SN}}=10^{51} \mathrm{ergs}$, corresponding to a relatively fast red-supergiant wind. It serves as demonstration of the stability of our results against the choice of hydrodynamical parameters that we even tested a Sedov solution, and the implications for the flux increase at a few $100 \mathrm{GeV}$ were virtually the same. The magnetic field in the precursor region is assumed to be $B_{\mathrm{p}}=23 \mu \mathrm{G}$, which is shock-compressed to a downstream field $B_{\mathrm{d}}=75 \mu \mathrm{G}$ inside the SNR (Ballet 2006; Acero et al. 2009).

RX J1713.7-3946 is considered to be $1600 \mathrm{yr}$ old, and we use that as fiducial age of RX J1713.7-3946. Fitting the Truelove-McKee profiles permits us to have a certain liberty in choosing the distance, the age, and the current shock velocity. The actual age is of minor relevance to the scenario at hand. The time span between two observations of the object and, as Eq. (10) illustrates, the shock velocity and the diffusion coefficient are important. The allowed range in current shock velocity is fairly small, thus setting limits on the age, and despite entering Eq. (10) quadratically, it does not lead to a large uncertainty in the timescale of flux variability. The location of the dense gas shell is prescribed by the necessity to reproduce the presently observed $\gamma$-ray spectrum from RX J1713.7-3946, as the precursor of cosmic rays at $10 \mathrm{TeV}$ should just reach it. That leaves the diffusion coefficient and, in particular, the efficacy of magneticfield amplification as the main sources of uncertainty, for which we use conservative assumptions.

We numerically solve the time-dependent cosmic-ray transport equation (Eq. (4)) using the above parameters (Telezhinsky et al. 2012a). We then calculate emission spectra and intensity maps at two epochs, at 1600 and at $1610 \mathrm{yr}$, including pion-decay (Huang et al. 2007), inverse Compton (IC) and synchrotron emission in turbulent magnetic field (Pohl et al. 2015). The dense gas shell radially extends from $8.75 \mathrm{pc}$ to $9.05 \mathrm{pc}$ from the SNR center, and so the shock does not interact with the dense material, but CRs in the shock precursor can efficiently reach the dense gas. A small separation between the gas shell and the forward shock is necessary to reproduce the $v F_{v}$-peak at $\sim 1 \mathrm{TeV}$ in the hadronic $\gamma$-ray emission. The results of our calculations are presented at Fig. 6 (solid red line). The HE $\gamma$-ray spectrum at the age of $1600 \mathrm{yr}$ is normalized to fit HESS data and to reconstruct the measured flux, $F=(17.2 \pm 0.4) \times 10^{-12}$ photons $\mathrm{cm}^{-2} \mathrm{~s}^{-1}$ (Aharonian et al. 2011).

The parameters are defined by the $\gamma$-ray flux ratio between the $\mathrm{TeV}$ band and the GeV band as well as the location of the peak in the $v F_{v}$ spectrum. The latter determines the separation between the forward shock and the gas shell, somewhat depending on the scaling of the diffusion coefficient in the precursor. The former prescribes the mass or gas density in the shell, $\sim 300 M_{\odot}$ and $n_{\mathrm{H}} \simeq 35 \mathrm{~cm}^{-3}$ for our SNR expansion model with $17 M_{\odot}$ of swept-up gas in the remnant. This is simply the amount of target material needed to fit the observed $\gamma$-ray spectrum; a thorough study of the hydrodynamical circumstances that could give rise to a shell with the required mass is beyond the scope of 


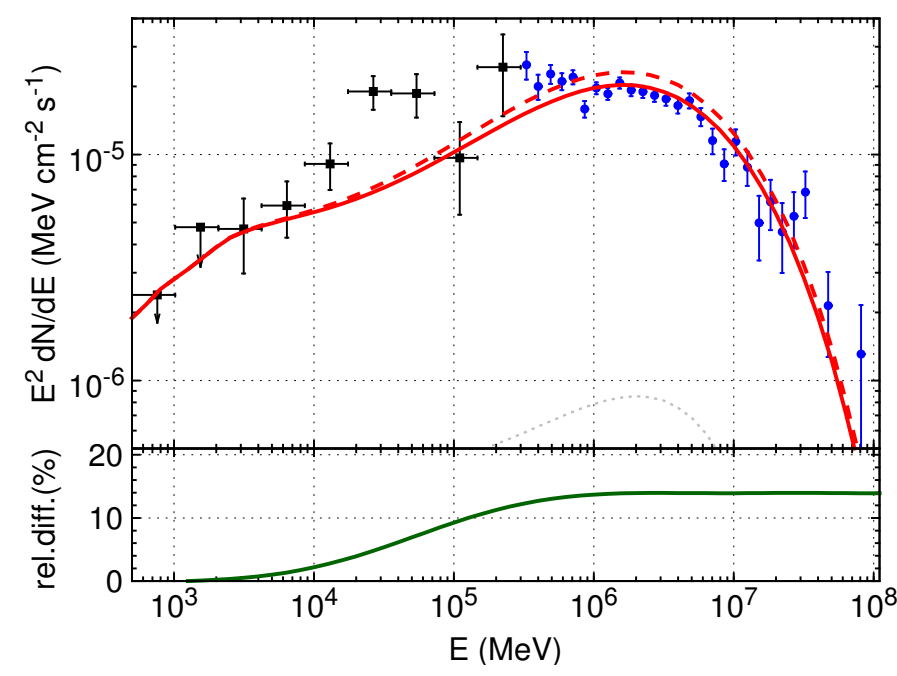

Fig. 6. Broadband $\gamma$-ray spectrum of RX J1713.7-3946 as measured with LAT (our results, black points) and with HESS (blue points), compared to calculated HE $\gamma$-ray spectra at the age of $1600 \mathrm{yr}$ (solid red line) and $1610 \mathrm{yr}$ (dashed red line). The IC contribution to both spectra is indicated for additional information (gray dotted line). The relative difference between spectra at two epochs is shown in the lower panel (solid green line).

this paper. The IC contribution is constrained by the observed flux of synchrotron X-rays and our choice of magnetic-field strength. Reproducing the spectral width of the $\gamma$-ray signal in the $v F_{v}$ spectrum shown in Fig. 5 requires a diffusion coefficient well in excess of the Bohm limit. In other words, the parameter $\eta \approx 10$ in Eq. (11), increasing with distance from the shock, and hence with $B=23 \mu \mathrm{G}$ and $\dot{r}_{\mathrm{s}}=4200 \mathrm{~km} \mathrm{~s}^{-1}$, we expect $\tau \simeq 100 \mathrm{yr}$ as timescale of variation. The numerical treatment indicates a flux increase of $15 \%$ over ten years, consistent with the analytical estimate.

The main prediction of our model thus is a secular increase with time of the HE $\gamma$-ray flux that is largely independent of the choice of parameters, provided they permit reproducing the peak in the $v F_{v}$ spectrum at $\sim 1 \mathrm{TeV}$ for the current size and expansion rate of RX J1713.7-3946. Figure 6 (dashed red line) shows the evolution of the $\gamma$-ray over $10 \mathrm{yr}$. The relative increase is about $15 \%$ at around $500 \mathrm{GeV}$ (see the green line at Fig. 6). The IC flux does not change during this period because the amount of target photons interacting with electrons in the shock precursor does not change noticeably. Also the change in morphology is too small to be detectable with CTA.

The calculated flux in the $1-100 \mathrm{TeV}$ energy band at the age of $1600 \mathrm{yr}$ amounts to $F_{1600}=17.2 \times 10^{-12}$ photons $\mathrm{cm}^{-2} \mathrm{~s}^{-1}$, compatible with that measured with HESS, whereas ten years later the flux would be $F_{1610}=19.9 \times 10^{-12}$ photons $\mathrm{cm}^{-2} \mathrm{~s}^{-1}$, with most of the increase coming from the shell. This change in the flux could be potentially detected with the CTA (Acharya et al. 2013). The systematic uncertainties in the flux scale for the original measurement with HESS are probably too large for that, and so one would need CTA data taken at least ten years apart.

\section{Conclusions}

In this work we analyzed more than five years of FermiLAT observations of the SNR RX J1713.7-3946, using highresolution $\gamma$-ray background maps that account for the HI selfabsorption. RX J1713.7-3946 appears as circular source with radius $r \simeq 0.55^{\circ}$ that is detected at $13 \sigma$ level in the energy range between $500 \mathrm{MeV}$ and $300 \mathrm{GeV}$. We see a tendency in the $\mathrm{GeV}$ band for the same intensity enhancement in the northwest region of the remnant that is seen with HESS and probably due to particles interacting with denser gas located there. The measured spectrum of RX J1713.7-3946 indeed shows a hard spectral index of $\gamma=1.53 \pm 0.07$ with an integral flux above $500 \mathrm{MeV}$ of $F=(5.5 \pm 1.1) \times 10^{-9}$ photons $\mathrm{cm}^{-2} \mathrm{~s}^{-1}$.

Phenomenological fits of the broadband SED demonstrate that in simple one-zone models for particle acceleration and emission it is hard to accommodate any pion-decay scenario. These fits also show that a common particle power-law spectrum with an exponential cutoff for electrons and protons faces some difficulty in simultaneously accounting for the X-ray, $\mathrm{GeV} \gamma$-ray, and $\mathrm{TeV} \gamma$-ray observations. A more complex model with additional parameters and more flexible assumptions could modify the shape of the broadband spectrum and give a better description of the $\gamma$-ray data.

We discussed alternative hadronic scenarios and pointed out some difficulties with them. In particular, we introduced a model of hadronic emission from RX J1713.7-3946 that reproduces the hard $\mathrm{GeV}$ spectrum with index $\gamma \simeq 1.5$ as emission from a shell of dense gas that is located a short distance upstream of the forward shock. The existence of this shell may be related to the winds of the progenitor star. Although this hadronic scenario is somewhat speculative, it has the benefit of being testable: we predict the hadronic $\gamma$-ray flux to increase at the level of $15 \%$ over ten years, which should be observable with the future CTA facility that is projected to operate for more than ten years (Acharya et al. 2013). We have verified that the growth in $\gamma$-ray flux is largely independent of the choice of parameters, provided they reproduce a $v F_{v}$-peak at $\sim 1 \mathrm{TeV}$. Whereas our model assumes spherical symmetry, real SNR are not that simple, and conditions are not the same in different regions. The stability of our result suggests that such a variation of parameters does not significantly reduce the secular increase in $\gamma$-ray flux.

A second implication of the scenario is that, within the next $50 \mathrm{yr}$, the forward shock of RX J1713.7-3946 should hit the gas shell, which would then light up in X rays and permit unprecedented studies of shock-cloud interactions. We stress that similar intensity fluctuations are expected in models invoking the forward shock moving through a medium in which most of the mass is organized in dense gas clouds.

Acknowledgements. Part of this work was supported by the Helmholtz Alliance for Astroparticle Physics HAP funded by the Initiative and Networking Fund of the Helmholtz Association. V.V.D.'s research on the high energy emission from young SNRs is supported by NASA Fermi grant NNX12A057G.

\section{References}

Abdo, A. A., Ackermann, M., Ajello, M., et al. 2011, ApJ, 734, 28 Acero, F., Ballet, J., Decourchelle, A., et al. 2009, A\&A, 505, 157 Acharya, B. S., Actis, M., Aghajani, T., et al. 2013, Astropart. Phys., 43, 3 Ackermann, M., Ajello, M., Allafort, A., et al. 2012a, ApJ, 755, 22 Ackermann, M., Ajello, M., Albert, A., et al. 2012b, ApJS, 203, 4 Aharonian, F., Akhperjanian, A. G., Aye, K.-M., et al. 2004, Nature, 432, 75 Aharonian, F., Akhperjanian, A. G., Bazer-Bachi, A. R., et al. 2011, A\&A, 531, $\mathrm{C} 1$

Atwood, W., Abdo, A. A., Ackermann, M., et al. 2009, ApJ, 697, 1071 Ballet, J. 2006, Adv. Space Res., 37, 1902

Bell, A. R. 1978, MNRAS, 182, 147

Cassam-Chenaï, G., Decourchelle, A., Ballet, J., et al. 2004, A\&A, 427, 199

Drury, L. O. 1983, Rep. Prog. Phys., 46, 973

Ellison, D. C., \& Bykov, A. M. 2011, ApJ, 731, 87

Gabici, S., \& Aharonian, F. A. 2014, MNRAS, 445, L70 
S. Federici et al.: Analysis of GeV-band $\gamma$-ray emission from supernova remnant RX J1713.7-3946

Gabici, S., Aharonian, F. A., \& Casanova, S. 2009, MNRAS, 396, 1629 Gibson, S. J., Taylor, A. R., Higgs, L. A., Brunt, C. M., \& Dewdney, P. E. 2005, ApJ, 626, 214

Huang, C.-Y., Park, S.-E., Pohl, M., \& Daniels, C. 2007, Astropart. Phys., 27, 429

Inoue, T., Yamazaki, R., Inutsuka, S.-i., \& Fukui, Y. 2012, ApJ, 744, 71

Kalberla, P. M., Burton, W., Hartmann, D., et al. 2005, A\&A, 440, 775

Koyama, K., Kinugasa, K., Matsuzaki, K., et al. 1997, PASJ, 49, L7

Mattox, J., Bertsch, D., Chiang, J., et al. 1996, ApJ, 461, 396

McClure-Griffiths, N. M., Dickey, J., Gaensler, B., et al. 2005, ApJS, 158, 178

McKee, C. F., van Buren, D., \& Lazareff, B. 1984, ApJ, 278, L115

Nolan, P. L., Abdo, A. A., Ackermann, M., et al. 2012, ApJS, 199, 31

Pfeffermann, E., \& Aschenbach, B. 1996, in International Conference on X-ray Astronomy and Astrophysics: Röntgenstrahlung from the Universe, 267
Pohl, M., Englmaier, P., \& Bissantz, N. 2008, ApJ, 677, 283

Pohl, M., Wilhelm, A., \& Telezhinsky, I. 2015, A\&A, 574, A43

Ressler, S. M., Katsuda, S., Reynolds, S. P., et al. 2014, ApJ, 790, 85

Rettig, R., \& Pohl, M. 2012, A\&A, 545, A47

Sano, H., Fukuda, T., Yoshiike, S., et al. 2015, ApJ, 799, 175

Slane, P., Gaensler, B. M., Dame, T., et al. 1999, ApJ, 525, 357

Strong, A., Porter, T., Digel, S., et al. 2010, ApJ, 722, L58

Tanaka, T., Uchiyama, Y., Aharonian, F. A., et al. 2008, ApJ, 685, 988

Telezhinsky, I., Dwarkadas, V., \& Pohl, M. 2012a, Astropart. Phys., 35, 300

Telezhinsky, I., Dwarkadas, V. V., \& Pohl, M. 2012b, A\&A, 541, A153

Telezhinsky, I., Dwarkadas, V. V., \& Pohl, M. 2013, A\&A, 552, A102

Truelove, J. K., \& McKee, C. F. 1999, ApJS, 120, 299

Uchiyama, Y., Aharonian, F., \& Takahashi, T. 2003, A\&A, 400, 567 